\title{
«César. Le Rhône pour mémoire » : une visite d'exposition entre raison et sentiment
}

"Cesar. The Rhône river as a memory-bank ": an exhibition visit between reason and emotion

\section{Fabienne Chameroy et Lucien Véran}

\section{(2) OpenEdition \\ Journals}

Édition électronique

URL : http://journals.openedition.org/ocim/1065

DOI : 10.4000/ocim.1065

ISSN : 2108-646X

Éditeur

OCIM

Édition imprimée

Date de publication : 1 mai 2012

Pagination : 33-38

ISSN : 0994-1908

Référence électronique

Fabienne Chameroy et Lucien Véran, « « César. Le Rhône pour mémoire » : une visite d'exposition entre raison et sentiment », La Lettre de l'OCIM [En ligne], 141 | 2012, mis en ligne le 01 mai 2014, consulté le 30 avril 2019. URL : http://journals.openedition.org/ocim/1065; DOI : 10.4000/ocim.1065 


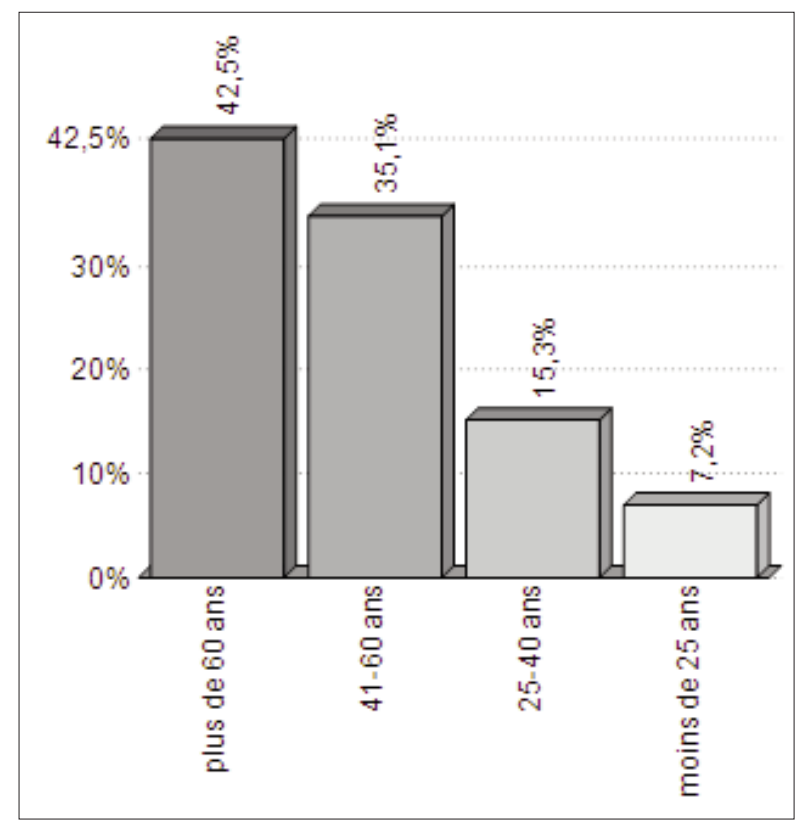

Répartition des visiteurs par classes d'âge

important des plus de 60 ans en région ProvenceAlpes-Côte d'Azur (25\% versus $22 \%$ au niveau national) et la proportion plus grande des femmes dans cette tranche d'âge peuvent expliquer en partie ces proportions. Ainsi, les femmes de plus de 60 ans représentent $28 \%$ de la population en région PACA. Il est intéressant de noter que, dans le public de l'exposition, le poids des plus de 60 ans passe de 42,5\% en moyenne à $27 \%$ au mois d'août. Le profil des visiteurs en pleine saison touristique est clairement plus jeune que celui du reste de l'année.

Sur l'ensemble, 66,1\% des visiteurs ont un niveau d'études supérieur au bac, et près de $30 \%$ sont diplômés du 3 e cycle.
Les retraités constituent la partie principale des visiteurs inactifs ${ }^{(4)}$, retraités donc ou personnes au foyer qui représentent à eux tous 50,1\% du public. Sur la base d'une répartition complète du public en 4 catégories : retraités et autres inactifs, enseignants (primaire, secondaire, supérieur), professions intermédiaires et supérieures (commerçants, professions libérales, cadres, cadres supérieurs) et professions diverses (artistes, employés, ouvriers) l'on obtient $24 \%$ pour les professions intermédiaires et supérieures, $13 \%$ d'enseignants et $12,8 \%$ de professions diverses. Plus que le métier c'est donc l'absence de métier et le temps dégagé par cette absence qui rend disponible pour la visite.

Les visiteurs, pour $82 \%$ d'entre eux, fréquentent d'autres musées. Cependant, 71,5\% sont des primo-visiteurs du MDDA, chiffre très nettement supérieur à celui de $50 \%$ constaté lors de précédentes expositions au musée (Vitalbo et Jacobi, 2003) ce qui confirme le pouvoir d'attraction spécifique de l'exposition.

Enfin, c'est principalement par la télévision et par l'émission «Des Racines et des Ailes » que les visiteurs ont été informés de la tenue de l'exposition. Près de $60 \%$ des visiteurs sont venus à l'exposition en étant informés par la télévision (cumul des citations de l'émission «Des Racines et des Ailes » et des autres émissions et journaux télévisés). Cette influence exceptionnelle place la télévision comme premier moyen de communication cité, loin devant les relations interpersonnelles habituellement première source d'information citée ${ }^{(5)}$.

\section{Raison et sentiment}

Une forte proportion des visiteurs est bien informée et emploie son temps libre à se cultiver. Accumulant

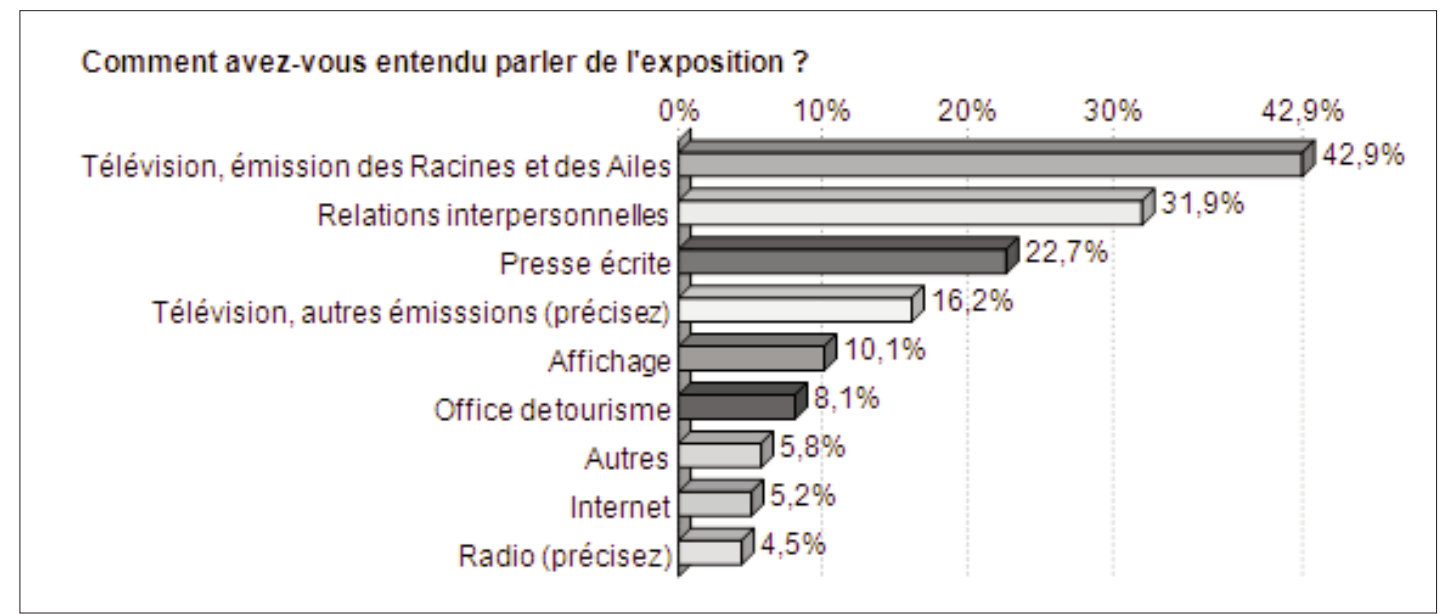

Modes d'informations des visiteurs 
de l'information il devient « expert en visite » donnant une orientation cognitive à son expérience sans exclure, s'il est sollicité pour cela, la recherche d'une émotion esthétique qui peut être provoquée par les œuvres et/ou la scénographie de l'exposition.

\section{Un public connaisseur}

Trois manières de mesurer le niveau d'expertise du visiteur ont été intégrées dans le questionnaire. La première consistait à mesurer l'expertise par une échelle d'auto-évaluation déjà développée par d'autres dans le cadre d'une visite d'exposition d'art moderne (Passebois et Aurier, 2004) et que nous avons adaptée. Le deuxième critère était celui de la lecture d'une revue d'art, d'archéologie ou d'histoire (nous avons demandé aux visiteurs de nous citer la revue). Le troisième critère était celui de la pratique muséale (rare, régulière, forte). Nous avons ainsi pu répartir les individus en 3 groupes que nous nommons les experts, les connaisseurs et les novices. Croiser ces trois critères permet de mettre un peu plus en lumière une caractéristique clef du public de l'exposition, son fort niveau moyen en matière de capital culturel. Seuls $17 \%$ des visiteurs sont au niveau bas des trois échelles et plus de $80 \%$ d'entre eux viennent consolider un savoir préalable que l'on peut considérer comme un des moteurs essentiels de leur déplacement.

La pratique muséale des novices est plus faible que celle des deux autres catégories (une visite de musée dans les cinq dernières années), les moins de 25 ans y sont surreprésentés et ils ne sont pas abonnés à une revue d'art, d'histoire ou d'archéologie. Leur niveau d'études est plus bas que les deux autres catégories et l'adjectif le plus fréquent utilisé pour qualifier leur ressenti de l'exposition est « désorienté ». Leurs attentes sont centrées sur les œuvres les plus médiatisées : le buste de Jules César et les œuvres découvertes dans le Rhône «vues à la télévision ».

La pratique muséale des connaisseurs est régulière (une visite d'un autre musée dans l'année) mais ils ne sont pas abonnés à une revue d'archéologie, d'art

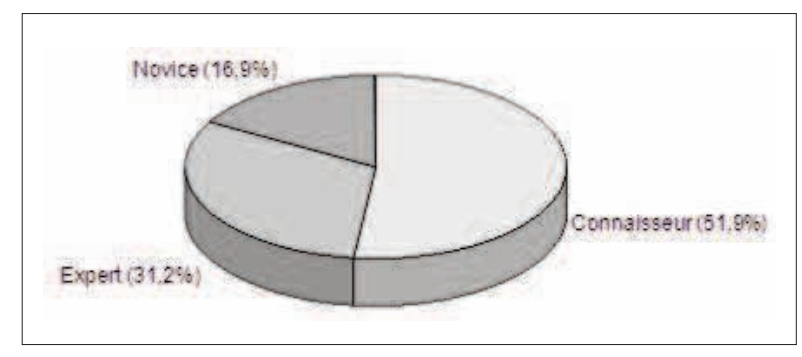

Niveau d'expertise du visiteur

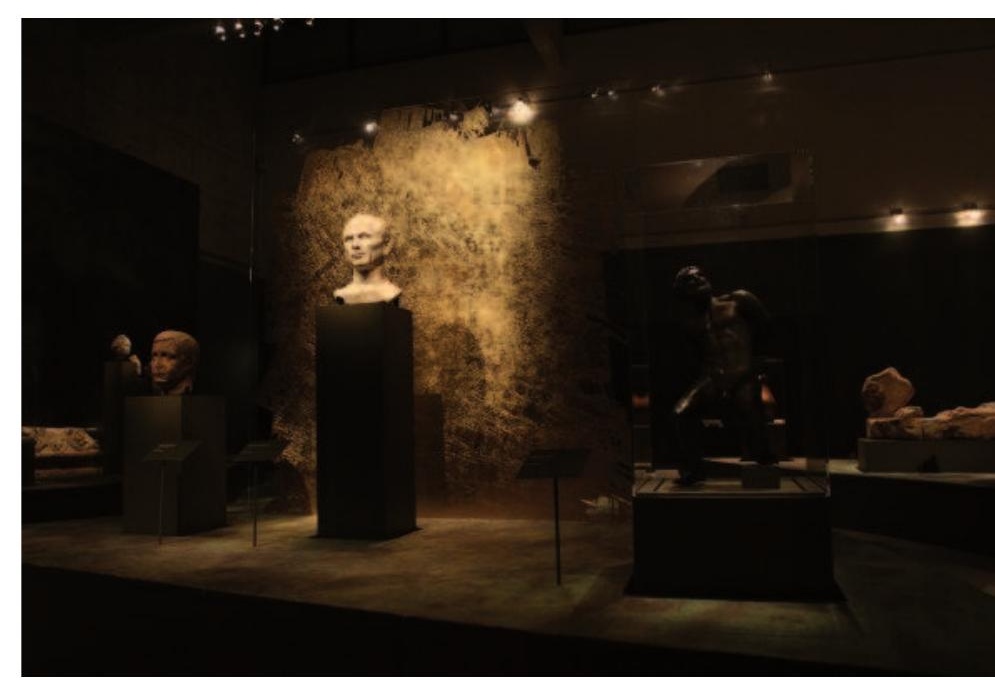

Le buste de César dans l'exposition (c) CG13/Serge Ben Lisa

ou d'histoire. Les professions libérales y sont surreprésentées. Elles ont une claire tendance à vouloir transmettre des messages et des informations à leur entourage. Ils représentent plus de $50 \%$ des visiteurs de l'exposition.

Les experts viennent plutôt par goût pour l'antiquité, l'art ou l'archéologie, ils sont plutôt abonnés à une revue d'art ou d'histoire ou d'archéologie, ils donnent une note maximum lorsqu'on les interroge pour savoir s'ils recommanderaient l'exposition. Ils préparent davantage leur visite que les autres catégories. Leurs attentes sont fortement dictées par leur goût pour l'Antiquité et sont relativement plus dirigées vers les œuvres elles-mêmes que sur l'ambiance, la scénographie et les éléments périphériques de l'exposition.

\section{Un public sensible}

L'analyse des appréciations fournies par les personnes interrogées permet de proposer un autre classement des visiteurs. Une deuxième typologie a été réalisée à partir de la notation des visiteurs sur trois types de questions portant sur leur appréciation détaillée de l'exposition : une dimension technique d'une part (éléments de mise en scène, confort de visite, déroulement...) une dimension de stimulation cognitive et enfin une dimension de stimulation ludique ou distractive. Certains visiteurs ont ainsi une appréciation plus émotionnelle que raisonnée de l'expérience vécue lors de leur visite. Nous avons procédé à une analyse en composantes principales (ACP) qui permet de résumer l'information transmise par l'évaluation détaillée en deux axes principaux et de classer les visiteurs. 


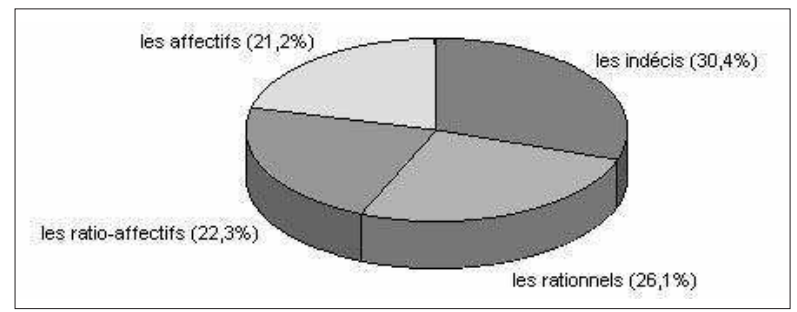

Typologie en fonction de l'appréciation de la visite

Quatre groupes archétypaux se distinguent alors: - les « affectifs » ont particulièrement apprécié la dimension émotionnelle et distractive de l'exposition, ils ont ressenti beaucoup de plaisir à la découvrir et ont vécu une expérience unique. Ils ont particulièrement apprécié les éléments de mise en scène des œuvres. Les «affectifs » ont été plus gênés par l'affluence que les autres catégories de visiteurs ;

- les « rationnels » sont dans une approche plus technique de la visite et s'intéressent surtout au contenu informatif de l'exposition. Ils sont plus intéressés par les œuvres que par les éléments de mise en scène ou périphériques, les éléments de stimulation cognitive ont été très appréciés par eux ;

- les « ratio-affectifs » sont sensibles à la fois comme les « rationnels » à la stimulation cognitive provoquée par l'exposition (s'enrichir, découvrir) et comme les «affectifs » à la charge émotionnelle que comporte la visite. Il y a davantage de femmes dans cette catégorie et de visiteurs venus deux fois à l'exposition ;

- les « indécis » qui ne valorisent ni l'enrichissement de leurs connaissances ni la recherche d'une émotion esthétique et ont une appréciation plus modérée de l'exposition représentent $30 \%$ des visiteurs. Les « indécis » ont plutôt critiqué la mise en scène des œuvres.

Les « rationnels » et « ratio-affectifs » ont une meilleure appréciation générale de l'exposition que les «affectifs » et les «indécis » et ils la recommanderaient davantage autour d'eux. Ce sont les «indécis » qui sont finalement les plus critiques, comme s'ils attendaient que les ordonnateurs de l'exposition leur fournissent un moyen de structurer leurs préférences et qu'ils leur reprochent donc de ne pas l'avoir fait.

Il est à souligner qu'en croisant les deux typologies, davantage d'experts s'avèrent être des " rationnels » alors qu'ils sont sous-représentés chez les «indécis » ce qui confirme une intuition. Les connaisseurs en revanche sont surreprésentés chez les « indécis » et sousreprésentés chez les « rationnels ». Les experts, connaisseurs ou novices peuvent tout autant être des « ratio-affectifs » que des « affectifs ». La raison n'exclut donc pas systématiquement ici le sentiment.
Cela peut être pris comme une bonne nouvelle et inciter les ordonnateurs à continuer à s'intéresser plus encore à l'équilibre délicat entre émotion et cognition dans la présentation des œuvres.

\section{Conclusion}

L'exposition César. Le Rhône pour mémoire a été un succès en termes de fréquentation et de satisfaction. Près de 390000 visiteurs sont venus sur une période de 15 mois au musée départemental de l'Arles Antique (MDAA) voir en priorité le buste de Jules César et neuf visiteurs sur dix se sont déclarés satisfaits de leur visite. Selon une expression fréquemment utilisée par les visiteurs il s'agissait de : «le voir » et l'on venait pour : «le buste».

Le public de l'exposition était classiquement composé en majorité de femmes érudites, retraitées, venant accompagnées et ayant une pratique muséale régulière. Le public était aussi principalement constitué de connaisseurs et d'experts (85\% des visiteurs) appréciant à la fois la dimension de stimulation cognitive et émotionnelle de l'exposition. Nul n'était besoin pour eux de choisir entre raison et sentiments, leur plaisir d'apprendre était réel et ils étaient très satisfaits de l'exposition. Ce sont les experts qui étaient les plus enclins à vouloir recommander l'exposition autour d'eux, la recommandation interpersonnelle (le bouche à oreille dans un vocabulaire plus commun) étant le deuxième mode d'information sur la tenue de l'exposition. Les novices, visiteurs plus jeunes et moins diplômés, sensibles aux éléments d'environnement des œuvres comme la scénographie et l'affluence, étaient les plus critiques. Ces novices se sont souvent sentis « désorientés » par l'exposition.

Les résultats présentés ici sont très sensiblement différents de ceux des études antérieures menées sur les publics du MDAA concernant les sources d'information citées par les visiteurs et concernant le volume observé de primo visiteurs. L'influence de la télévision a été exceptionnelle. L'émission spéciale de la série «Des Racines et des Ailes » sur France 3 a été le premier mode d'information cité (pour près de 1 visiteur sur 2) et a façonné le niveau d'attente des visiteurs. L'exposition enfin et fait exceptionnel a su attirer une part de nouveaux visiteurs de $70 \%$.

\section{Notes}

(1) Chiffres clefs, DEPS, 2011.

(2) Muséostat, Fréquentation des musées de France, ministère de la Culture et de la Communication, 2009. 
(3) Plus précisément et avec un risque d'erreur de $5 \%$ la proportion de femmes se situe entre $57,9 \%$ et $67,1 \%$.

(4) L'on définit ici conventionnellement les inactifs comme : « Les personnes qui ne sont ni en emploi ni au chômage : jeunes de moins de 15 ans, étudiants, retraités, hommes et femmes au foyer, personnes en incapacité de travailler ». (source Insee : www.insee.fr/fr/methodes/default.asp? page $=$ definitions/liste-definitions.htm)

(5) Vitalbo, V. et Jacobi, D. op. cit. p. 6. Information par les « connaissances » : $50 \%$.

\section{Bibliographie}

Chiffres clefs, statistiques de la culture-Musées, ministère de la Culture et de la Communication, département des Études de la Prospective et des Statistiques (DEPS), La Documentation française, 2011.
Donnat, O. Pratiques culturelles 1973-2008. Dynamiques générationnelles et pesanteurs sociales. Paris : ministère de la Culture et de la Communication, DEPS, Collection « Culture Études », 2011.

Passebois, J. et Aurier, P. Le rôle de l'expertise des consommateurs dans l'expérience culturelle; une approche par la valeur de consommation. 9es journées de Recherche en Marketing de Bourgogne, Dijon, novembre 2004.

Vitalbo, V. et Jacobi, D. Musée de l'Arles et de la Provence antiques. Les publics des expositions, La mort n'est pas une fin (15 septembre 2002-5 janvier 2003) et D'un monde à l'autre: Naissance d'une chrétienté en Provence IVe-VIe siècles (15 septembre 2001-6 janvier 2002). Avignon : Université d'Avigon, Laboratoire Culture et Communication, 2003. 